\title{
Switching of Conducting Planes by Partial Dimer Formation in $\mathrm{IrTe}_{2}$
}

\author{
Tatsuya ToriYama ${ }^{1}$, Masao Koвori ${ }^{1}$, Takehisa Konishi ${ }^{2}$, Yukinori Oнta ${ }^{1 *}$, Kunihisa Sugimoto $^{3}$, Jungeun \\ $\mathrm{KIm}^{3}$, Akihiko FuJiwara ${ }^{3}$, Sunseng Pyon ${ }^{4,5}$, Kazutaka Kudo ${ }^{5}$, and Minoru Nohara ${ }^{5 \dagger}$ \\ ${ }^{1}$ Department of Physics, Chiba University, Chiba 263-8522, Japan \\ ${ }^{2}$ Graduate School of Advanced Integration Science, Chiba University, Chiba 263-8522, Japan \\ ${ }^{3}$ Japan Synchrotron Radiation Research Institute (JASRI), SPring-8, Hyogo 679-5198, Japan \\ ${ }^{4}$ Department of Applied Physics, The University of Tokyo, Tokyo 113-8656, Japan \\ ${ }^{5}$ Department of Physics, Okayama University, Okayama 700-8530, Japan
}

(Received September 15, 2013)

\begin{abstract}
Single-crystal X-ray diffraction was employed to study the structural-electronic phase transition of $\operatorname{IrTe}_{2}$ at approximately $270 \mathrm{~K}$. The low-temperature structure was found to be a triclinic (space group $P \overline{1}$ ) characterized by the partial formation of $\mathrm{Ir}_{2}$ dimers in the triangular lattice of $\mathrm{IrTe}_{2}$, resulting in a structural modulation with a wave vector of $\boldsymbol{q}=(1 / 5,0,-1 / 5)$. First-principles band calculations demonstrate that tilted two-dimensional Fermi surfaces emerge in the triclinic phase, suggesting that switching of the conducting planes occurs from the basal plane of the trigonal $\operatorname{IrTe}_{2}$ to the tilted plane normal to $\boldsymbol{q}$ of the triclinic $\mathrm{IrTe}_{2}$.
\end{abstract}

Making and breaking chemical bonds in a solid often results in a drastic change in the physical properties and thus offers useful ways of controlling the electronic states; examples includes the metal-insulator transition, ${ }^{1-5}$ superconductivity, ${ }^{6-11}$ and the magnetic quantum critical point. ${ }^{12}$ For instance, the formation of arsenic dimers that occurs in the adjacent FeAs layers of iron-based superconductors results in a two-dimensional to three-dimensional change in the topology of the Fermi surface, which in turn leads to the loss of the iron magnetic moment and thus the high-transition-temperature superconductivity. ${ }^{6,7}$ In this Letter, we report that the partial formation of iridium dimers in the triangular lattice of $\mathrm{IrTe}_{2}$ results in the emergence of dimerized $\mathrm{Ir}_{2}$ and non-dimerized Ir layers that are stacked alternately along a structural modulation vector of $\boldsymbol{q}=(1 / 5,0,-1 / 5)$. First-principles band calculations demonstrate that the partial formation of iridium dimers results in tilted two-dimensional Fermi surfaces, thus switching the conducting planes from the basal plane of the trigonal $\mathrm{IrTe}_{2}$ at high temperatures to the plane normal to $\boldsymbol{q}$ of the triclinic $\mathrm{IrTe}_{2}$ at low temperatures.

$\mathrm{IrTe}_{2}$ crystallizes in a trigonal $\mathrm{CdI}_{2}$-type structure with the space group $P \overline{3} m 1$ ( $\# 164)$. Edge-sharing $\operatorname{IrTe}_{6}$ octahedra form two-dimensional $\mathrm{IrTe}_{2}$ layers that are stacked along the $c$ axis, as shown schematically in Figs. 1(a) and 1(b). In each layer, the Ir atoms form a regular triangular lattice with a uniform Ir-Ir bond length of $3.925 \AA$. This compound undergoes a first-order structural phase transition at approximately $270 \mathrm{~K} .{ }^{13}$ Matsumoto et al. ${ }^{13}$ concluded that the average structure below $270 \mathrm{~K}$ is monoclinic with the space group $C 2 / m(\sharp 12)$ in which the Ir-Ir bond length along the monoclinic $b$-axis is uniformly reduced so that the regular triangular lattice is deformed into an isosceles triangular lattice.

*E-mail: ohta@faculty.chiba-u.jp

†E-mail: nohara@science.okayama-u.ac.jp
Recently, transmission electron microscopy experiments have revealed that the structural phase transition is accompanied by the evolution of non-sinusoidal structural modulation of $\boldsymbol{q}=(1 / 5,0,-1 / 5),{ }^{14}$ indicating that the structural phase transition is not of a simple charge-density-wave (CDW) type. An optical spectroscopy study suggested that the structural phase transition is driven by a reduction in the kinetic energy of the electrons due to Te $5 p$ band splitting below the transition temperature. ${ }^{15} \mathrm{X}$-ray photoemission spectroscopy ${ }^{16}$ and angle-resolved photoemission spectroscopy (ARPES) ${ }^{17}$ have indicated the importance of the orbital degeneracy of Ir $5 d$ and/or Te $5 p$ for the transition. Very recently, Oh et al. ${ }^{14}$ suggested that the structural phase transition involves the depolymerization-polymerization of anionic Te bonds. Interestingly, suppression of the structural phase transition by chemical doping or intercalation results in the emergence of superconductivity at up to $3.1 \mathrm{~K} .{ }^{18-22}$ Thus, it is important to determine the low-temperature crystal structure to elucidate the structural-electronic phase transition mechanism of $\operatorname{IrTe}_{2}$.

Single crystals of $\mathrm{IrTe}_{2}$ were grown using the self-flux technique. ${ }^{15,23} \mathrm{~A}$ mixture of Ir and Te powder at an atomic ratio of 18:82 was placed in an alumina crucible that was sealed in an evacuated small quartz tube and then placed into a larger quartz tube. The larger quartz tube was evacuated and sealed, and the mixture was initially heated to $950^{\circ} \mathrm{C}$; this temperature was maintained for $10 \mathrm{~h}$. The temperature was then increased slowly to $1160^{\circ} \mathrm{C}$, before the mixture was cooled to $900^{\circ} \mathrm{C}$ at a rate of $1-2^{\circ} \mathrm{C} / \mathrm{h}$. The quartz tube was then quenched in ice water after decantation of the Te flux. The obtained crystals exhibited a phase transition at approximately $270 \mathrm{~K}$ as determined by resistivity and magnetization measurements. ${ }^{23}$

Synchrotron radiation X-ray diffraction measurements were performed on beamline BL02B1 at SPring-8, Japan, us- 
Table I. Crystallographic data of $\operatorname{IrTe}_{2}$ at 300 and $20 \mathrm{~K}$.

\begin{tabular}{lll}
\hline \hline Formula & \multicolumn{2}{c}{$\mathrm{IrTe}_{2}$} \\
Formula weight & \multicolumn{2}{c}{447.42} \\
\hline Temperature $(\mathrm{K})$ & 300 & 20 \\
Space group & $P \overline{3} m 1(\sharp 164)$ & $P \overline{1}(\sharp 2)$ \\
$a(\AA)$ & $3.92530(10)$ & $3.94860(10)$ \\
$b(\AA)$ & $3.92530(10)$ & $6.64480(10)$ \\
$c(\AA)$ & $5.39520(10)$ & $14.4045(3)$ \\
$\alpha\left({ }^{\circ}\right)$ & 90 & $80.245(6)$ \\
$\beta\left(^{\circ}\right)$ & 90 & $87.404(6)$ \\
$\gamma\left({ }^{\circ}\right)$ & 120 & $72.511(5)$ \\
Volume $\left(\AA^{3}\right)$ & $71.992(3)$ & $355.252(13)$ \\
$Z$ & 1 & 5 \\
$R_{\text {int }}$ & 5.79 & 6.57 \\
\hline \hline
\end{tabular}

ing monochromatized X-ray with an energy of $35.05 \mathrm{keV}$ $(\lambda=0.354 \AA)$. Measurements were performed at 300 and $20 \mathrm{~K}$ using a cryogenic He-flowing system (XR-HR10KS, Japan Thermal Engineering Co. Ltd.). A cylindrical imaging plate with a camera length of $191.3 \mathrm{~mm}$ was adopted. ${ }^{24}$ At $T=300$ and $20 \mathrm{~K}$, diffraction images in a scattering vector range of up to approximately $34 \AA^{-1}$ were taken every 13 and 24 frames, respectively, at an exposure time of 1 min per image. The oscillation angle of the crystal $(\omega)$ in each frame was $15^{\circ}$. Typical diffraction images are shown in Fig. 2. Data were collected and processed using the RAPIDAUTO program (Rigaku) and were corrected for Lorentz and polarization effects. The structures were solved by direct methods (SHELXS-97) and expanded using Fourier techniques. All the atoms were refined anisotropically (SHELXL97). The final cycle of the full-matrix least-squares refinement was based on aall data, $I>2 \sigma(I)\}=\{261,261\}$ and $\{11716,10472\}$ observed reflections for $T=300$ and 20 $\mathrm{K}$, respectively. Unweight and weighted agreement factors of $R=\sum\left\|F_{0}|-| F_{c}\right\| / \sum\left|F_{0}\right|, R_{1}=\sum\left\|F_{0}|-| F_{c}\right\| / \sum\left|F_{0}\right|$ $\left(F_{0}>4 \sigma\left(F_{0}\right)\right)$, and $w R_{2}=\left[\sum\left(w\left(F_{0}^{2}-F_{c}^{2}\right)^{2} / \sum w\left(F_{0}^{2}\right)^{2}\right]^{1 / 2}\right.$ were used. The $R, R_{1}$, and $w R_{2}$ values were $\{0.0179,0.0179$, $0.0478\}$ and $\{0.0754,0.0694,0.242\}$ for $T=300$ and $20 \mathrm{~K}$, respectively. The crystal structure of the triclinic phase $(T=$ $20 \mathrm{~K}$ ) was refined as a twin-domain crystal using the TWIN and BASF commands of SHELXL-97. The final composition ratio of the twin-domain crystal was $0.712(1): 0.288(1)$. Crystallographic data and fractional coordinates obtained at both temperatures are summarized in Table 1 and 2, respectively.

Figures 1(c) and 1(d) show the low-temperature crystal structure of $\mathrm{IrTe}_{2}$ determined at $20 \mathrm{~K}$. The structure is triclinic with the space group $P \overline{1}(\sharp 2)$. The low-temperature structure is characterized by three crystallographically independent iridium sites, denoted as Ir1, Ir2, and Ir3 in Fig. 1(d). The bond lengths of adjacent iridium atoms along the trigonal $a$-axis are modulated considerably: The Ir3-Ir3 bond length of $3.069 \AA$ is considerably shorter than the Ir1-Ir2 and Ir2-Ir3 bond lengths (3.943 and $4.027 \AA$, respectively), which results in a non-sinusoidal structural modulation along the trigonal $a$-axis that is in accordance with the electron diffraction mea- (a)

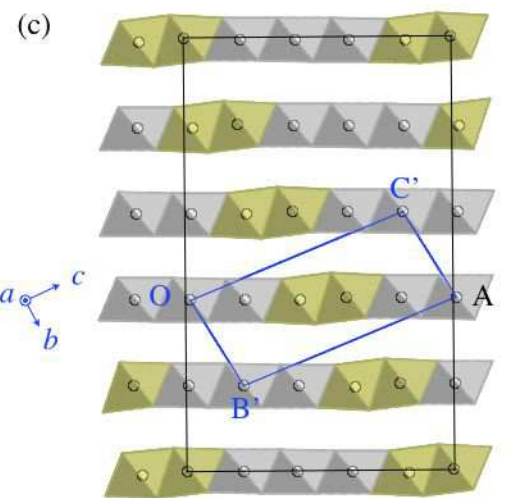

(b)

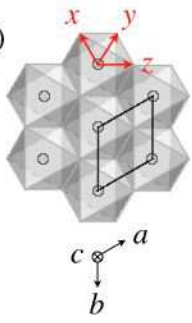

Fig. 1. (Color online) (a) and (b) The crystal structure of $\mathrm{IrTe}_{2}$ at $300 \mathrm{~K}$. Black lines represent the unit cell of the trigonal lattice. (c) and (d) The crystal structure of $\mathrm{IrTe}_{2}$ at $20 \mathrm{~K}$. Blue lines represent the unit cell of the triclinic lattice. Black lines represent the $5 a \times b \times 5 c$ supercell $(a, b$, and $c$ are the high-temperature trigonal-cell parameters).
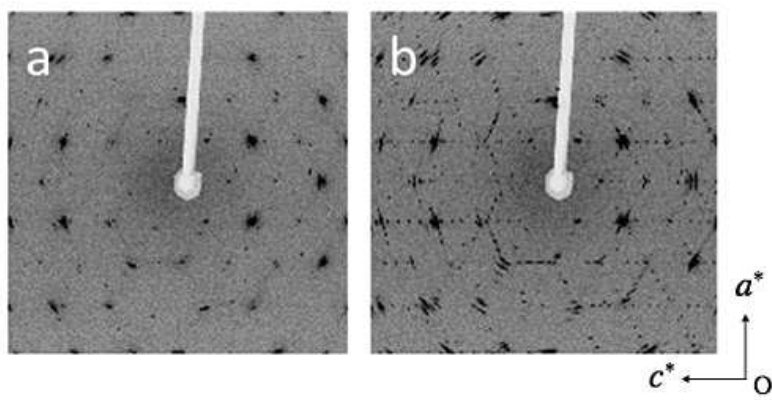

Fig. 2. Typical diffraction images of $\mathrm{IrTe}_{2}$ taken at (a) $300 \mathrm{~K}$ and (b) $20 \mathrm{~K}$.

surements. ${ }^{14,19}$ The Ir3-Ir3 bond length is comparable to that of the dimerized $\operatorname{Ir}(3.012 \AA)$ of $\mathrm{CuIr}_{2} \mathrm{~S}_{4} \cdot{ }^{4}$ Thus, the structural phase transition of $\operatorname{IrTe}_{2}$ can be viewed as the partial formation of a single $\mathrm{Ir}_{2}$ dimer from the five iridium atoms along the trigonal $a$-axis. The average bond length is compatible with the average structure. ${ }^{13}$ There is no structural modulation along the trigonal $b$-axis, and a uniform Ir bond length of $3.949 \AA$ is observed. Thus, along the trigonal $b$-axis direction there are stripes of dimerized Ir 3 and non-dimerized Ir 1 and Ir2. The position of these stripes shifts to $\pm \boldsymbol{a}$ along the trigonal $a$-axis in the adjacent layers, as shown in Fig. 1(c), 
Table II. Atomic coordinates and thermal displacement parameters of $\mathrm{IrTe}_{2}$ at 300 and $20 \mathrm{~K}$.

\begin{tabular}{lllll}
\hline \hline \multicolumn{5}{c}{$300 \mathrm{~K}$} \\
\hline site & $x / a$ & $y / b$ & $z / c$ & $U_{\text {iso }}$ \\
Ir1 & 0 & 0 & 0 & $0.01047(7)$ \\
Te1 & $1 / 3$ & $2 / 3$ & $0.25305(4)$ & $0.01110(7)$ \\
\hline \hline \multicolumn{5}{r}{$20 \mathrm{~K}$} \\
site & $x / a$ & $y / b$ & $z / c$ & $U_{\text {iso }}$ \\
Ir1 & 0 & 0 & 0 & $0.00356(6)$ \\
Ir2 & $0.36069(5)$ & $0.21315(3)$ & $0.203394(15)$ & $0.00357(6)$ \\
Ir3 & $-0.29000(6)$ & $0.43031(4)$ & $0.411623(16)$ & $0.00360(6)$ \\
Te1 & $-0.00026(11)$ & $-0.05426(7)$ & $0.18477(3)$ & $0.00381(7)$ \\
Te2 & $0.36127(11)$ & $0.27091(6)$ & $0.01679(3)$ & $0.00377(7)$ \\
Te3 & $0.35701(10)$ & $0.15939(6)$ & $0.38782(3)$ & $0.00385(7)$ \\
Te4 & $-0.27774(11)$ & $0.48038(7)$ & $0.22267(3)$ & $0.00386(7)$ \\
Te5 & $0.08129(10)$ & $0.70076(6)$ & $0.41086(3)$ & $0.00386(7)$ \\
\hline \hline
\end{tabular}

resulting in a structural modulation of $\boldsymbol{q}=(1 / 5,0,-1 / 5)$.

Modulation of the Te-Te distances appears in the triclinic phase, but it is much smaller than that of the Ir-Ir distances. In the high-temperature trigonal phase, there are three characteristic Te-Te distances: $3.498 \AA$ between adjacent $\mathrm{IrTe}_{2}$ layers, $3.548 \AA$ between the upper and lower Te atoms within an $\mathrm{IrTe}_{2}$ layer, and $3.925 \AA$ between the in-plane Te atoms of an $\mathrm{IrTe}_{2}$ layer. The short Te-Te bond between adjacent $\mathrm{IrTe}_{2}$ layers has been discussed previously, and was shown to result in direct covalent Te-Te bonds and a Te $5 p$ contribution to the electronic density of states at the Fermi level. ${ }^{25}$ In the low-temperature phase, a large modulation of 3.429-4.115 was observed in the in-plane Te-Te distance, while modulations in the other Te-Te distances were smaller (3.368-3.547 $\AA$ between adjacent $\operatorname{IrTe}_{2}$ layers and 3.488-3.561 ̊ between the upper and lower Te atoms in an $\operatorname{IrTe}_{2}$ layer). The shortest Te-Te bond length of $3.368 \AA$ in the triclinic phase of $\mathrm{IrTe}_{2}$ is much longer than that observed in the modulated structure of $\mathrm{AuTe}_{2}$ with a distorted $\mathrm{CdI}_{2}$-type structure $(2.88 \AA) .{ }^{10,26}$ The smaller modulations of the Te bond lengths suggest a dominant Ir $5 d$ contributes to the structural-electronic phase transition mechanism of $\mathrm{IrTe}_{2}$, although there is a finite Te $5 p$ contribution through the large mixing between the $\operatorname{Ir} 5 d$ and Te $5 p$ bands.

To study the change in the electronic states across the structural phase transition, we carried out electronic structure calculations using the full-potential augmented plane-wave and local orbital methods, as implemented in the WIEN2k code. ${ }^{27}$ We applied the generalized gradient approximation for electron correlations and considered the exchange-correlation part of the potential using the Perdew-Burke-Ernzerhof exchangecorrelation functional. ${ }^{28}$ Spin-orbit coupling (SOC) was taken into account for both Ir and Te. The maximum modulus of the reciprocal vectors $K_{\max }$ and muffin-tin radii of the atoms $R_{\mathrm{MT}}$ were chosen such that $R_{\mathrm{MT}} K_{\max }=7$. The lattice parameters and atomic coordinates were taken from our experimental data, and the Brillouin zone of the trigonal phase (triclinic phase) was sampled using a $16 \times 16 \times 10(14 \times 8 \times 3) k$ mesh. (a)

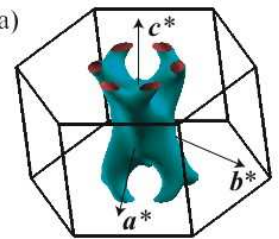

(c)
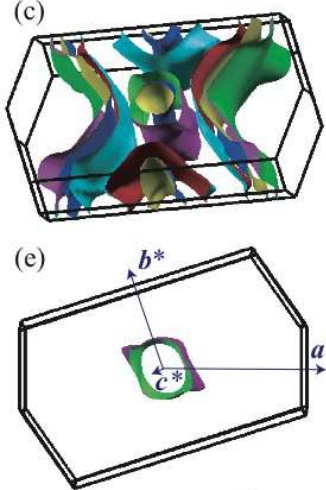

(g)

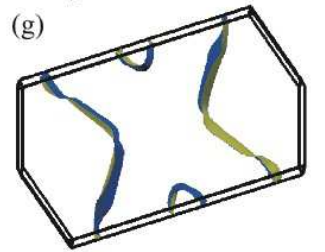

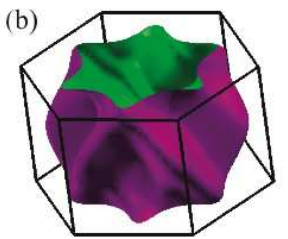

(d)
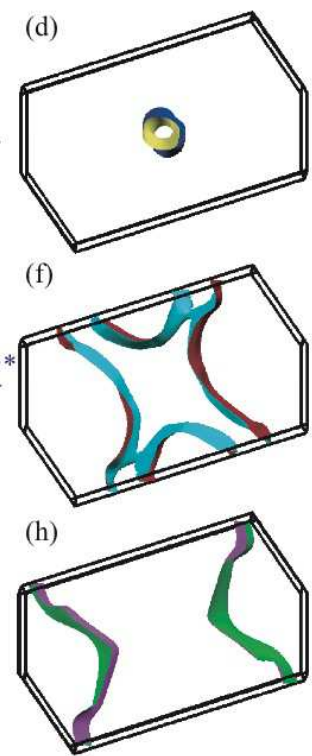

Fig. 3. (Color online) Fermi surfaces calculated for the [(a) and (b)] hightemperature trigonal phase and [(c)-(h)] low-temperature triclinic phase of $\mathrm{IrTe}_{2}$. All the Fermi surfaces in the low-temperature phase are summarized in (c) and are illustrated separately in (d)-(h). The reciprocal lattice vectors $\left(\boldsymbol{a}^{*}, \boldsymbol{b}^{*}, \boldsymbol{c}^{*}\right)$ are shown in (e). In each Fermi-surface sheet, the contributions come predominantly from (d) Te3 and Te5; (e) Te1, Te2, and Te4; (f) Ir1, Ir2, Te1, Te2, and Te4; (g) Ir1, Ir2, Te1, Te2, and Te4; and (h) Ir3 and Te5.

The calculated band dispersion, width, and Fermi surfaces of the high-temperature trigonal phase agree with those determined by the angle-resolved photoemission spectroscopy, ${ }^{16}$ indicating that the Hubbard-type repulsive interaction $U$ is negligible in $\operatorname{IrTe}_{2}$. The finite $U$ in the GGA $+U^{29,30}$ scheme does not affect the basic features of the state near the Fermi level, thus the shape of the Fermi surface. No spin-polarized solutions were found, consistent with the absence of CurieWiess behavior. ${ }^{18}$

Figure 3 shows the calculated Fermi surfaces of the hightemperature trigonal and low-temperature triclinic phases of $\mathrm{IrTe}_{2}$. In the high-temperature phase, shown in Figs. 3(a) and (b), the Fermi surfaces are rather three dimensional and electronic quasi-two-dimensionality occurs in the basal $a b$ plane of the trigonal lattice [see Figs. 1(a) and (b)]. In the low-temperature phase, however, it is immediately clear in Figs. 3(c)-(h) that all five Fermi-surface sheets exhibit a fair quasi-two-dimensionality consisting of small cylinderlike surfaces and strongly warped quasi-one-dimensional-like sheets, the direction of which indicates that the quasi-twodimensional conducting planes are now parallel to the $a b$ plane of the triclinic lattice [see Figs. 1(c) and (d)] or normal 

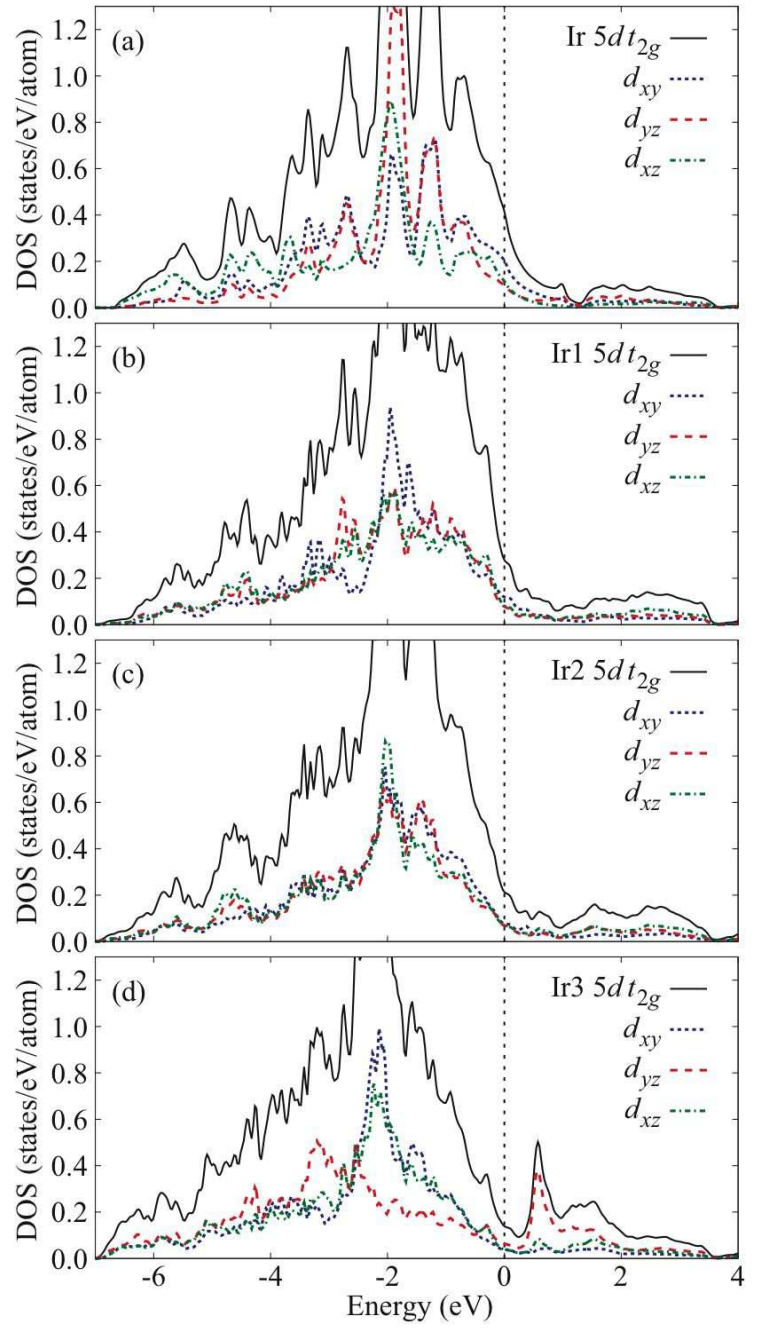

Fig. 4. (Color online) Orbital-decomposed partial DOS calculated for (a) the high-temperature trigonal phase and (b)-(d) the low-temperature triclinic phase of $\mathrm{IrTe}_{2}$. The $d_{x y}, d_{y z}$, and $d_{x z}$ components in the $t_{2 g}$ manifold of the Ir $5 d$ bands are shown for (b) Ir1, (c) Ir2, and (d) Ir3. The vertical line represents the Fermi level.

to the structural modulation vector $\boldsymbol{q}=(1 / 5,0,-1 / 5)$. This change in the Fermi surfaces demonstrates that switching of the conducting planes occurs from the basal plane of the trigonal $\mathrm{IrTe}_{2}$ to the tilted plane normal to $\boldsymbol{q}$ of the triclinic $\mathrm{IrTe}_{2}$ as well as the enhancement of two-dimensionality across the structural phase transition at approximately $270 \mathrm{~K}$. The Fermi surfaces calculated using the average monoclinic structure with uniform Ir-Ir bonds for the low-temperature phase exhibit neither switching of conducting planes nor dimensional crossover. ${ }^{15,20}$ Thus the partial formation of Ir dimers is crucial in switching the conducting planes and enhancing two dimensionality in the low-temperature triclinic phase of $\mathrm{IrTe}_{2}$, reminiscent of the formation of As dimers in iron-based superconductors, which results in a dimensional crossover. ${ }^{6,7}$

Ir and Te are subject to strong SOC; the band exhibits a large spin-orbit (SO) splitting of the order of $1 \mathrm{eV} .{ }^{20} \mathrm{How}-$ ever, the SOC seems not to be involved in the mechanism of the structural phase transition of $\mathrm{IrTe}_{2}$. Figure 4 shows the orbital-decomposed partial DOS calculated for the hightemperature trigonal and low-temperature triclinic phases of $\operatorname{IrTe}_{2}$, where the $d_{x y}, d_{y z}$, and $d_{x z}$ components in the $t_{2 g}$ manifold of the Ir $5 d$ bands are illustrated [see Figs. 1(b) and (d) for the definition of the coordinate axes $(x, y, z)]$. Compared with the partial DOS for the high-temperature phase, a striking change occurs in the $d_{y z}$ component in the $t_{2 g}$ manifold of the dimerized Ir3 atoms [Fig. 4(d)]. The three $t_{2 g}$ components are mostly occupied by electrons in the high-temperature phase [Fig. 4(a)], but the formation of $\mathrm{Ir}_{2}$ dimers in the low-temperature phase results in strong bonding-antibonding splitting in the $d_{y z}$ bands of the two Ir3 atoms, which increases the energy of the antibonding bands above the Fermi level [note the peak at $\sim 0.7 \mathrm{eV}$ in Fig. 4(d)] and decreases the energy of the bonding bands by $\sim 1 \mathrm{eV}$ [note the peaks at $\sim-3 \mathrm{eV}$ in Fig. 4(d)]. The splitting is by far larger than the SO splitting, ${ }^{20}$ suggesting that lowering in energy of the $d_{y z}$ bonding orbital plays an important role in the mechanism of the structural phase transition of $\mathrm{IrTe}_{2} \cdot{ }^{31}$ This is analogous to $\mathrm{CuIr}_{2} \mathrm{~S}_{4}$, in which orbital ordering takes place at the structural phase transition. ${ }^{4,32}$ The weight of the DOS is thus largely depleted around the Fermi level at the dimerized Ir3 sites. The partial DOS for the $p_{z}$ component of the $5 p$ bands of Te 5 located between the dimerized Ir3 atoms also undergoes a similar change near the Fermi level (results not shown here). Thus total DOS is reduced from 1.89 to 0.97 states/eV/IrTe 2 , consistent with the reduction of magnetic susceptibility and electronic specific heat. ${ }^{13,15,18}$ The local DOSs at the Ir3 and Te5 sites are thus strongly reduced at the Fermi level, although a band gap does not appear, indicating that the dimerized $\mathrm{Ir}_{2}$ planes become less conducting or effectively disconnect the conducting planes. In contrast, the planes in which the Ir1 and Ir2 atoms are located remain highly conducting as the height of the local DOS at the Fermi level suggests, yielding the quasi-two-dimensional conducting planes normal to the modulation vector $\boldsymbol{q}=(1 / 5,0,-1 / 5)$. Such switching of the conducting planes that occurs with the structural phase transition is, to the best of our knowledge, quite a rare example in transition-metal compounds.

In summary, we have employed single-crystal X-ray diffraction to study the structural-electronic phase transition of $\mathrm{IrTe}_{2}$ at approximately $270 \mathrm{~K}$. The low-temperature triclinic structure (space group $P \overline{1}$ ) is characterized by the partial formation of $\mathrm{Ir}_{2}$ dimers that occurs in the triangular lattice of $\mathrm{IrTe}_{2}$, resulting in a structural modulation with a wave vector of $\boldsymbol{q}=(1 / 5,0,-1 / 5)$. First-principles band calculations have demonstrated that tilted two-dimensional Fermi surfaces emerge in the triclinic phase of $\mathrm{IrTe}_{2}$, suggesting that switching of the conducting planes occurs from the basal plane of the trigonal $\mathrm{IrTe}_{2}$ to the tilted plane normal to $\boldsymbol{q}$ of the triclinic $\mathrm{IrTe}_{2}$.

Part of this work was performed at the Advanced Science Research Center, Okayama University. The study was partially supported by a Grant-in-Aid for Scientific Research (C) 
(No. 25400372) and for Young Scientists (B) (No. 24740238) from the Japan Society for the Promotion of Science (JSPS) and the Funding Program for World-Leading Innovation R\&D on Science and Technology (FIRST Program) from JSPS. The synchrotron radiation experiments performed at SPring- 8 were supported by the Japan Synchrotron Radiation Research Institute (JASRI; Proposal Nos. 2011B1072, 2012B1463, 2012B1055). T.T. acknowledges support from the JSPS Research Fellowship for Young Scientists.

1) A. Cavalleri, Th. Dekorsy, H. H. W. Chong, J. C. Kieffer, and R. W. Schoenlein, Phys. Rev. B 70, 161102(R) (2004).

2) M. W. Haverkort, Z. Hu, A. Tanaka, W. Reichelt, S. V. Streltsov, M. A. Korotin, V. I. Anisimov, H. H. Hsieh, H.-J. Lin, C. T. Chen, D. I. Khomskii, and L. H. Tjeng, Phys. Rev. Lett. 95, 196404 (2005).

3) N. Katayama, M. Uchida, D. Hashizume, S. Niitaka, J. Matsuno, D. Matsumura, Y. Nishihata, J. Mizuki, N. Takeshita, A. Gauzzi, M. Nohara, and H. Takagi, Phys. Rev. Lett. 103, 146405 (2009).

4) P. G. Radaelli, Y. Horibe, M. J. Gutmann, H. Ishibashi, C. H. Chen, R. M. Ibberson, Y. Koyama, Y.-S. Hor, V. Kiryukhin, and S.-W. Cheong, Nature 416, 155 (2002).

5) D. Hirai, M. Bremholm, J. M. Allred, J. Krizan, L. M. Schoop, Q. Huang, J. Tao, and R. J. Cava, Phys. Rev. Lett. 110, 166402 (2013).

6) S. Kasahara, T. Shibauchi, K. Hashimoto, Y. Nakai, H. Ikeda, T. Terashima, and Y. Matsuda, Phys. Rev. B 83, 060505(R) (2011).

7) M. Danura, K. Kudo, Y. Oshiro, S. Araki, T. C. Kobayashi, and M. Nohara, J. Phys. Soc. Jpn. 80, 103701 (2011).

8) D. Hirai, F. von Rohr, and R. J. Cava, Phys. Rev. B 86, 100505(R) (2012).

9) S. R. Saha, N. P. Butch, T. Drye, J. Magill, S. Ziemak, K. Kirshenbaum, P. Y. Zavalij, J. W. Lynn, and J. Paglione, Phys. Rev. B 85, 024525 (2012).

10) K. Kudo, H. Ishii, M. Takasuga, K. Iba, S. Nakano, J. Kim, A. Fujiwara, and M. Nohara, J. Phys. Soc. Jpn. 82, 063704 (2013).

11) G. Cao, T. Furubayashi, H. Suzuki, H. Kitazawa, T. Matsumoto, and Y. Uwatoko, Phys. Rev. B 64, 214514 (2001).

12) S. Jia, P. Jiramongkolchai, M. R. Suchomel, B. H. Toby, J. G. Checkelsky, N. P. Ong, and R. J. Cava, Nat. Phys. 7, 207 (2011).

13) N. Matsumoto, K. Taniguchi, R. Endoh, H. Takano, and S. Nagata, J.
Low Temp. Phys. 117, 1129 (1999).

14) Y. S. Oh, J. J. Yang, Y. Horibe, and S.-W. Cheong, Phys. Rev. Lett. 110, 127209 (2013)

15) A. F. Fang, G. Xu, T. Dong, P. Zheng, and N. L. Wang, Sci. Rep. 3, 1153 (2013).

16) D. Ootsuki, Y. Wakisaka, S. Pyon, K. Kudo, M. Nohara, M. Arita, H. Anzai, H. Namatame, M. Taniguchi, N. L. Saini, and T. Mizokawa, Phys. Rev. B 86, 014519 (2012).

17) D. Ootsuki, S. Pyon, K. Kudo, M. Nohara, M. Horio, T. Yoshida, A. Fujimori, M. Arita, H. Anzai, H. Namatame, M. Taniguchi, N. L. Saini, and T. Mizokawa, J. Phys. Soc. Jpn. 82, 093704 (2013).

18) S. Pyon, K. Kudo, and M. Nohara, J. Phys. Soc. Jpn. 81, 053701 (2012)

19) J. J. Yang, Y. J. Choi, Y. S. Oh, A. Hogan, Y. Horibe, K. Kim, B. I. Min, and S.-W. Cheong, Phys. Rev. Lett. 108, 116402 (2012).

20) M. Kamitani, M. S. Bahramy, R. Arita, S. Seki, T. Arima, Y. Tokura, and S. Ishiwata, Phys. Rev. B 87, 180501(R) (2013).

21) K. Kudo, M. Kobayashi, S. Pyon, and M. Nohara, J. Phys. Soc. Jpn. 82, 085001 (2013)

22) A. Kiswandhi, J. S. Brooks, H. B. Cao, J. Q. Yan, D. Mandrus, Z. Jiang, and H. D. Zhou, Phys. Rev. B 87, 121107(R) (2013).

23) S. Pyon, K. Kudo, and M. Nohara, Physica C 494, 80 (2013).

24) K. Sugimoto, H. Ohsumi, S. Aoyagi, E. Nishibori, C. Moriyoshi, Y. Kuroiwa, H. Sawa, and M. Takata, AIP Conf. Proc. 1234, 887 (2010).

25) S. Jobic, R. Brec, and J. Rouxel, J. Solid State Chem. 96, 169 (1992).

26) W. J. Schutte and J. L. de Boer, Acta Crystallographica Section B 44, 486 (1988)

27) P. Blaha, K. Schwarz, G. K. H. Madsen, D. Kvasnicka, and J. Luitz, WIEN2k (Technische Universität Wien, Austria, 2002).

28) J. P. Perdew, K. Burke, and M. Ernzerhof, Phys. Rev. Lett. 77, 3865 (1996).

29) V. I. Anisimov, I. V. Solovyev. M. A. Korotin, M. T. Czyżyk, and G. A. Sawatzky, Phys. Rev. B 48, 16929 (1993).

30) A. I. Lichtenstein, V. I. Anisimov, and J. Zaanen, Phys. Rev. B 52, R5467 (1995).

31) (Supplemental Material) See supplemental material for further details on the significance of the SOC of $\mathrm{IrTe}_{2}$.

32) D. I. Khomskii and T. Mizokawa, Phys. Rev. Lett. 94, 156402 (2005).

Note added in proof - We noticed a paper by Pascut et al. [arXiv:1309.3548, Phys. Rev. Lett. (in press)], reporting similar results. 